\title{
Research on the Evaluation of Agricultural Disaster Resistance in Henan Province Based on GIS Technology
}

\author{
Hong $\operatorname{Han}^{1}$, Guanghui Feng ${ }^{1, *}$, Guoqu Deng ${ }^{1}$ \\ ${ }^{1}$ School of Management, Henan University of Science and Technology, Luoyang, 471023, Henan, China
}

\begin{abstract}
Using the University of Notre Dame Global Climate Adaptation Index (ND-GAIN) assessment model for reference, the assessment system and model of agricultural disaster resistance in Henan Province are constructed.The spatial characteristics of agricultural disaster resistance subsystem (vulnerability and preparedness) of 18 cities in Henan Province in 2018 were studied by using GIS technology from six perspctives such as sensitivity, exposure, emergency, management, capacity to analyze. The results show that the agricultural disaster resistance ability of Henan Province is in the middle to lower level, showing the spatial difference pattern of high in the west, low in the southeast and weak in the north and south; the difference of vulnerability index is small, and the difference of preparedness index is large; the difference in the degree of preparedness is the main reason for the obvious difference in the level of agricultural disaster resistance; the development of agricultural disaster resistance in different regions is not balanced, and the disaster resistance in Zhengzhou is the strongest, followed by Luoyang and Sanmenxia, Zhoukou and Zhumadian are less resilient.
\end{abstract}

\section{Introduction}

Agricultural disasters have profoundly affected the social stability and healthy economic development of China for a long time. The core of disaster prevention and mitigation is to enhance the ability to resist risks, and the key is to build the ability to resist disasters. In May 2019, the United Nations convened the sixth Global Platform for Disaster Reduction conference on the theme "Resilient Dividends: Towards Sustainable and Inclusive Societies ", which set out the goal of building resilient societies that are sustainable and inclusive.

The Disaster Resilience study began with the concept of "resilience ". The American ecologist Holling creatively introduced resilience into ecosystem stability research in the $1970 \mathrm{~s}$, and ecosystem resilience research was more widely used in ecological environment restoration[1]; subsequently Timmerman and so on introduced resilience into the field of social and environmental change, linking resilience to vulnerability for the first time, believing resilience to be the system's ability to withstand and recover from external shocks[2]; joint national International Strategy for Disaster Reduction (UNISDR) introduced the concept of resilience into the field of disaster science as a measure of the ability of a system or community to resist or reduce the loss of natural disasters[3]. Based on a comprehensive consideration of the above scholars' views, Cutter points out that resilience includes both destructive outcomes that enable the organizational system to repair disasters and adaptive processes that improve the ability to respond to threats through the reorganization, transformation and learning of social organizational systems[4]. Resilience has also begun to gradually transition to resilience, research findings show that the two have basically converged. With the development of research, the ability to resist disasters has gone on Growing into a comprehensive concept that emphasizes both the intrinsic qualities of an organization or system and the ability to respond to and recover from disasters.

On the research of disaster resistance evaluation, scholars at home and abroad mainly focus on the following two aspects: first, qualitative evaluation. Qualitative evaluation pays more attention to the importance of resilience, influencing factors and intrinsic characteristics, and determines the resilience of society, organization or system through certain factor conditions. The National Research Council of the United States, in its report Resilience: A National Imperative, for example, in 2012, identified a unified framework of qualitative indicators for measuring resilience, namely,vulnerable populations, key environmental infrastructure, social factors and built infrastructure[5]; Bruneau identified resilience concept of necessity, and put forward that the evaluation of resilience to disasters is composed of four interrelated dimensional spaces: technical, organizational, social and economic[6]; Second, quantitative evaluation.

1Email: my16397@163.com; 2,"Email:1138292241@qq.com; ${ }^{3}$ Email:dengguoqu@haust.edu.cn 
Han Jiannan introduced the disaster resistance ability of integrated prevention and control system into the evaluation model from the inherent attributes of the traditional disaster body and the passive attributes of the disaster bearing body for quantitative research [7]. Shaw proposed the Climate Disaster Resilience Index , which uses weighted combined averages to calculate targets from five aspects: natural environment, physical factors, social, economic and infrastructure regional resilience index[8]. Therefore, this study draws lessons from the global climate adaptation index (ND-GAIN) model of Notre Dame University in the United States, constructs the evaluation index system of agricultural resilience in Henan Province, and analyzes the agricultural resilience strength, spatial zoning characteristics and main influencing factors of 18 cities in Henan Province in 2018 in combination with GIS technology, so as to provide a reference basis for improving the agricultural resilience of Henan Province and reducing the difference of agricultural resilience among regions.

\section{Evaluation methodology evaluation indicator system}

\subsection{Evaluation methodology}

The University of Notre Dame Global Climate Adaptability Index (referred to as ND-GAIN) is an emerging leading indicator at the national level for the prediction and prevention of climate change issues. It is an evaluation system developed by the University of Notre Dame to assess the resilience and adaptability of countries or regions to natural disasters[9]. It outlines a country's vulnerability to global challenges, such as climate change, and its willingness to increase resilience to natural disasters. ND-GAIN index model framework includes two aspects: one is the vulnerability of a country or region in the context of climate change, assessing the vulnerability of a country through six life support sectors, such as food supply, water safety and health; the other is the level of preparedness of a country or region to respond to natural disasters at the social level and in terms of economic capacity, measured mainly in terms of economic preparedness, management preparedness and social readiness, taking into account national development, social inequality and education. The index model aims to enhance the understanding of countries or regions around the world of climate change adaptation and resilience to disasters and to assist Decision makers make decisions.

\subsection{Evaluation indicators system}

\subsubsection{Construction of Evaluation Index System}

Agricultural resilience is a complex system, which requires a multi-faceted, multi-level and multi-angle scientific of regional agricultural resilience. There are many factors affecting agricultural resilience, both engineering and non-engineering. Based on the ND-GAIN evaluation model, the regional agricultural disaster resilience evaluation model is divided into two parts: agricultural disaster vulnerability index model and agricultural disaster preparedness index model. Vulnerability index is measured from three aspects: sensitivity, exposure and adaptability, and readiness index is measured from three aspects: socio-economic support ability, emergency management ability and agricultural engineering defense ability.Taking into account the actual situation of natural geography, hydrometeorology and agricultural environment in Henan Province, and referring to the research results of Zhang Hao, Shi Peijun and Gu Hongbo[10-15], the evaluation system of agricultural resilience in Henan Province has been constructed through the replacement and screening of a series of indicators, as shown in Table 1.

Table1. Evaluation index system of Agricultural Disaster Resistance

\begin{tabular}{|c|c|c|c|}
\hline Target layer & Criteria layer & Indicator layer & type \\
\hline \multirow{8}{*}{$\begin{array}{l}\text { Vulnerability index } \\
\text { (VI) }\end{array}$} & \multirow{3}{*}{ Sensitivity } & Water consumption per unit of cultivated land & - \\
\hline & & Proportion of rural population $\mathrm{X} 2$ & - \\
\hline & & Age factor & - \\
\hline & \multirow{3}{*}{ Exposure } & Food crop exposure ratio & - \\
\hline & & Agricultural economic density & - \\
\hline & & Annual precipitation & - \\
\hline & \multirow{3}{*}{ Adaptability } & Mechanical and electrical wellsper unit cultivated area & + \\
\hline & & Road network density & + \\
\hline \multirow{5}{*}{$\begin{array}{l}\text { Preparation Index } \\
\text { (PI) }\end{array}$} & & Water storage capacity & + \\
\hline & \multirow{2}{*}{ Economic support capacity } & Per capita disposable income in rural areas & + \\
\hline & & Disaster insurance & + \\
\hline & \multirow{2}{*}{$\begin{array}{c}\text { Emergency management } \\
\text { capacity }\end{array}$} & Medical and health assistance capacity & + \\
\hline & & Number of emergency managers & + \\
\hline
\end{tabular}


Information on Agricultural Disaster Prevention +

Agricultural engineering defence capabilities
Effective irrigation of cultivated land

Weather station density

Agricultural mechanization levelper unit cultivated area

\subsubsection{Evaluation Index Data Calculation and Standardization}

\section{1) Sources of data}

Taking 2018 as the evaluation year and 18 cities in Henan Province as the basic evaluation unit, considering the availability and practicability of the data, the data collection is mainly derived from the data collection. The data issued by the official departments, such as Henan Statistical Yearbook, Henan Water Resources Bulletin, Henan Rural Statistical Yearbook, and 2018 Henan Municipal Statistical Bulletin for National Economic and Social Development, are all standard statistical data issued by government departments.

2) Data standardization

Reference to the calculation formula of the global climate adaptability index of the university of mary, combined with the data characteristics of each evaluation index, this study used linear interpolation method to estimate and fill the missing data; in order to eliminate the impact of dimensional differences on the comprehensive evaluation, this study Min-Max standardized the original data.Set the $X_{i j}$ as the original index data,$X_{i j}^{\prime}$ as the index standardized data, the specific processing methods are as follows:

Standardized formula for positive indicators:

$$
X_{i j}^{\prime}=\left(X_{i j}-X_{i j \min }\right) /\left(X_{i j \max }-X_{i j \min }\right)
$$

Standardized formula for negative indicators:

$$
X_{i j}^{\prime}=\left(X_{i j \max }-X_{i j}\right) /\left(X_{i j \max }-X_{i j \min }\right)
$$

\section{3) Index weight calculation}

The entropy weight method is used to determine the weight of the evaluation index. Entropy is a measure of the degree of information disorder, the smaller the information entropy of the index, the greater the uncertainty, the greater the amount of information explained by the index, the greater the role played in the comprehensive evaluation, and the greater the weight[16]. Let the standardized value of the index be ${ }^{X_{i j}}$, entropy value is ${ }^{e_{j}}$, weight is ${ }^{W_{j}}$. The calculation steps are:

$$
e_{j}=-\ln (n)^{-1} \sum_{i=1}^{n} p_{i j} \ln p_{i j}
$$

$$
W_{j}=\left(1-e_{j}\right) /\left(n-\sum e_{j}\right)
$$

Of which $p_{i j}=X_{i j}^{\prime} / \sum_{i=1}^{n} X_{i j}^{\prime}, 0 \leq e_{j} \leq 1 \quad ; \quad$ if $p_{i j}=0$,then $\lim _{p_{i j} \rightarrow 0} p_{i j} \ln p_{i j}=0.0001$.

\subsubsection{Resilience Assessment Score Model}

Reference to the index calculation method in the ND-GAIN model, the second level index value corresponds to the sum of the weight coefficients of the third level index, and the first level index is the average value of the corresponding second level index. where the vulnerability index optimal score is 0 , the lower the better; the preparation degree index optimal score is 1 , the higher the better.

The vulnerability index model:

$$
V I=\sum\left(W_{j} X_{i j}^{\prime}\right) / n
$$

Preparation index model:

$$
P I=\sum\left(W_{j} X_{i j}^{\prime}\right) / n
$$

Agricultural resilience index model:

$$
N D-G A I N=(P I-V I+1) \times 50
$$

The $n$ is the corresponding number of secondary indicators, the ${ }^{W_{j}}$ is the third level index weight, the ${ }^{X_{i j}^{\prime}}$ is the standardized value of the index, the $P I$ is the readiness index, the $V I$ is the vulnerability index, and the $N D-G A I N$ is the resilience index.

\section{Resultsts and analysis}

\subsection{ND-GAIN score}

According to the disaster resilience evaluation model, the scores of disaster resilience index, vulnerability index and readiness index of Henan province in 2018 can be calculated, as shown in Table

Table2. Index of Agricultural Disaster Resistance of cities in Henan Province in 2018

\begin{tabular}{cccccccccc}
\hline Score & Zhengzhou & Luoyang & Sanmenxia & Jiaozuo & Kaifeng & Hebi & Nanyang & Xinxiang & Anyang \\
\hline VI & 0.38 & 0.43 & 0.426 & 0.44 & 0.55 & 0.47 & 0.52 & 0.436 & 0.45 \\
PI & 0.75 & 0.72 & 0.643 & 0.54 & 0.57 & 0.46 & 0.47 & 0.38 & 0.37 \\
ND-GAIN & 68.51 & 64.22 & 60.85 & 55.18 & 51.25 & 49.56 & 47.64 & 47.14 & 46.09 \\
\hline Score & Luohe & Xinyang & Pingdingshan & Puyang & Jiyuan & Shangqiu & Shangqiu & Zhoukou & Zhumadian \\
VI & 0.48 & 0.51 & 0.57 & 0.52 & 0.59 & 0.53 & 0.54 & 0.61 & 0.58
\end{tabular}




\begin{tabular}{cccccccccc} 
PI & 0.39 & 0.43 & 0.45 & 0.39 & 0.46 & 0.32 & 0.33 & 0.37 & 0.33 \\
ND-GAIN & 45.34 & 45.94 & 43.97 & 43.52 & 42.91 & 39.52 & 39.24 & 37.90 & 37.68 \\
\hline
\end{tabular}

In general, the evaluation index of agricultural resilience in Henan Province is low, and it shows the characteristics of high in the west, low in the southeast and weak in the north and south. Due to the influence of monsoon climate and different north and south latitudes, the difference between east and west terrain is obvious. The eastern and southeast plains are vulnerable to drought, strong winds and other disasters; the western and northern regions are vulnerable to extreme meteorological disasters such as wind and waterlogging, frost and hail, and the agricultural disaster resistance ability in Henan Province has obvious spatial differences. From the spatial region, Zhengzhou, Luoyang, Sanmenxia have the strongest resilience, followed by Kaifeng, Jiaozuo, mainly its agricultural disaster preparedness index score is relatively high; The weakest areas are Zhoukou, Zhumadian, Shangqiu and

other places, and the vulnerability of agricultural disasters is high, so it is urgent for local government organizations to effectively defend themselves and strengthen the building of agricultural resilience to disasters.

\subsection{Spatial zoning analysis of agricultural resilience subsystem}

Based on the ArcGis10.6 software, the paper presents a visual analysis of agricultural resilience in Henan province fromsixfactors: sensitivity, exposure, adaptability, socio-economic, support, emergency management and agricultural engineering defense. The natural breakpoint method is used to divide the influence factors of each subsystem in space, as shown in figure 1.

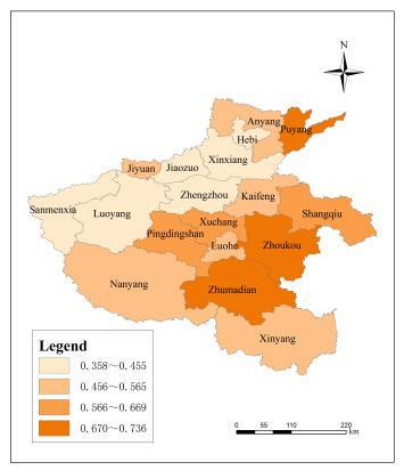

(a) Sensitivity factors

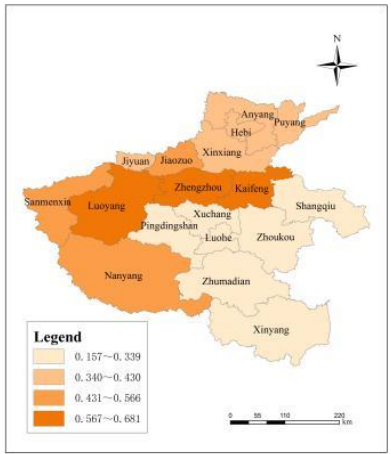

(d) Economic support capacity

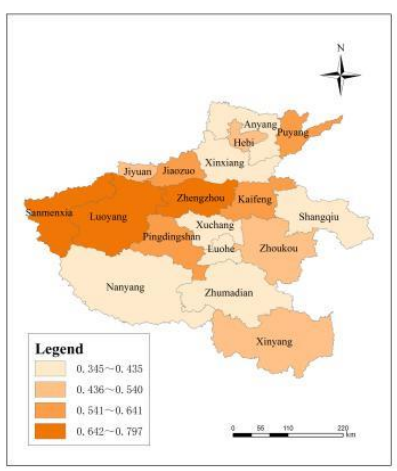

(b) Exposure factors

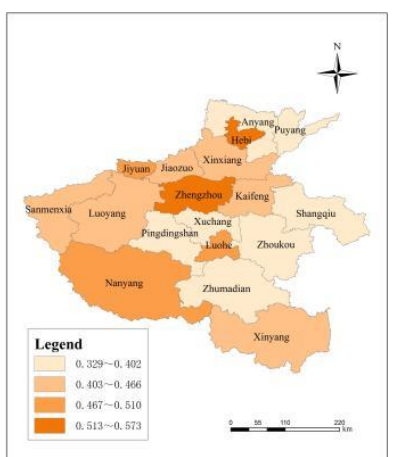

(e)Emergency management capacity

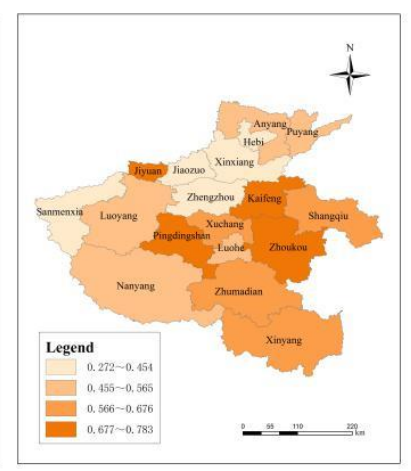

(c) Adaptive factors

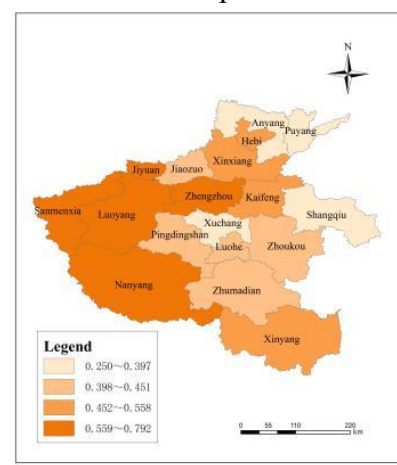

Figure1. Spatial distribution of influencing factors of agricultural disaster resistance in Henan Province

\subsubsection{Spatial distribution of vulnerability assessment subsystems}

Vulnerability assessment subsystem impact factors include sensitivity, exposure and adaptability. As shown in Figure 1 (a)、(b)、(c): the sensitivity and exposure of agricultural risk in the east and south of Henan Province are obviously higher than those in the west and north, except in Jiyuan City, while the adaptability is generally high in the west, low in the east and weak in the north and weak in the south, which is roughly consistent with the spatial distribution characteristics of sensitivity and exposure. On the whole, the vulnerability evaluation index of Henan Province is high and the difference is small. As far as the region is concerned, Zhoukou and Zhumadian are more sensitive and exposed to the risk of agricultural natural disasters, and respond to external pressure and self-regulation to recover the ability is insufficient, the vulnerability index is high, so the evaluation of the resilience of the two is at a low level; the sensitivity and exposure degree of Zhengzhou, Luoyang and Sanmenxia cities are all low, and the evaluation of adaptability is high, so the vulnerability index is low, and the level of agricultural resilience is relatively high. It shows that sensitivity and exposure under agricultural risk pressure are the short board to 
reduce agricultural vulnerability and improve agricultural resilience, and to improve local agricultural risk pressure according to the role of key impact factors of subsystem, which is also the preferred direction to enhance agricultural resilience in the future. But because the two are the inherent attributes of agricultural disaster resistance, it is difficult to measure accurately. Therefore, all regions should take active measures to improve agricultural adaptability, reduce vulnerability and achieve sustainable development of agricultural resources.

\subsubsection{Spatial distribution of the subsystem for evaluation of readiness}

The sub-system of preparation degree evaluation is mainly composed of three factors: social and economic support ability, emergency management ability and agricultural engineering defense ability. As shown in Figure 1 (d)、(e)、(f): spatially, the evaluation of preparedness in the central and western regions of Henan Province is significantly higher than that in the southeast region, and the overall distribution is uneven and the regional differentiation is more obvious, among which the emergency management ability is quite different from the north and south; from the regional point of view, Zhengzhou and Luoyang City are in the advantage in three aspects of social and economic support ability,emergency management ability, agricultural engineering defense ability, Kaifeng City has a large investment in agricultural disaster emergency management, Jiyuan City as a river. South province's only urban-rural integration demonstration area, agricultural scale, industrialization, in agricultural engineering defense capacity building is more prominent; but in the southeast of Zhumadian City, Xuchang City, Shangqiu City and Zhoukou City multi-plain, vulnerable to natural disasters such as drought, floods and other natural disasters, relatively low level of economic development, insufficient resources security, weak emergency management capacity, to a large extent affected the development of agricultural resilience. Therefore, it is necessary not only to speed up the development of agriculture's own economy, but also to attach importance to the construction of emergency management and other related systems, and to shift the agricultural disaster resistance from focusing only on the response and recovery after the outbreak of disasters to the extent possible.To prepare for the prediction, prevention and resilience of agricultural disaster risks, to implement disaster prevention and mitigation work in various sectors, to create a cultural atmosphere of public awareness of crisis prevention and to carry out public awareness campaigns on popular knowledge of emergency response, to scientifically and accurately assess agricultural risks and their own resilience to disasters, and to mobilize all sectors of society to actively support agriculture in disaster response and disaster relief, to strengthen the construction of agricultural disaster-resistant infrastructure, and to provide adequate preparations for regional agriculture in disaster prevention and protection.

\section{Conclusions}

Based on the global climate adaptation index (ND-GAIN) model of Notre Dame University in the United States, the agricultural resilience of 18 regions in Henan Province was evaluated by constructing the evaluation index system of agricultural resilience. The results show that:

The overall agricultural resilience of Henan Province is in the middle and lower level, the development of agricultural resilience is not balanced, the vulnerability index of each region is small, and the degree of preparation is obviously different. Vulnerability assessment subsystem is the inherent characteristics of agriculture, is the main impact factors of agricultural vulnerability; readiness assessment subsystem includes economic, social, natural and other complex attributes, socio-economic support capacity is the most fundamental guarantee for agricultural disaster prevention and mitigation. Agriculture in various regions is affected by economic, hydrological, geomorphological, climatic, soil and other factors, and the path focus on the improvement of disaster resistance is also different.

In space, the agricultural resilience of Henan Province presents the characteristics of high west, low southeast and weak north and south. Among them, the southeast region with high vulnerability and high preparation degree is mainly distributed in the central and western regions with relatively rapid economic development, such as Zhengzhou, Luoyang and Sanmenxia. The agricultural disaster resistance of 18 regions in Henan Province was ranked from strong to weak, and it was concluded that the areas with higher disaster resistance were Zhengzhou City, Luoyang City and Sanmenxia City, and the areas with lower disaster resistance were Zhoukou City, Xuchang City and Zhumadian City.

The difference in the level of preparedness among regions is the main factor affecting the marked difference in the level of resilience of agriculture, and the level of preparedness in one region determines the region's efficient response capacity and resilience to disasters. Therefore, the high level of resilience should not only reduce the exposure and sensitivity of the disaster-bearing body, but also do a good job of preparing for agricultural disaster resistance, from passive "impact" response to active self-assessment, defense, economic logistics support, emergency response management, agricultural engineering construction is indispensable.

Systematic and comprehensive evaluation index system and effective evaluation method are the basis and guarantee of agricultural resilience evaluation. agricultural production system is a complex system. In view of different agricultural situation, regional situation and disaster situation, different disaster prevention and mitigation management measures should be taken to construct a new effective prevention and adaptation 
mechanism. In addition, the comprehensive evaluation involves the discussion of weight value, evaluation value and distribution characteristic, this study only focuses on the analysis of evaluation value and distribution characteristic, and the weight of each influencing factor needs to be further explored.

\section{References}

1. Holling C S.Resilience and Stability of Ecological Systems[J].Annual Review of Ecology and Systematics,1993 (4):1-23.

2. Timmerman P. Vulnerability, Resilience and the Collapse of Society: A review of models and possibe climatic applications[J].Toronto: Institute for Environmental Studies, University of Toronto, 1981,4(1): 396-396.

3. Ge Yi, Shi Peijun, Xu Wei, et al. Advances and comments on the study of resilience [J].] Disaster Science ,2010,25(3):119-124 129.

4. Cutter S L,Barnes L,Berry M,et al. A Place-based Model for Understanding Community Resilience Tonatural Disasters[J]. Global environmental change,2008,18(4): 598-606.

5. Zeng microwave, Hou Tingting, Yang Cancan, et al. A Study on Agricultural Flood Disaster Assessment Method Based on Remote Sensing Monitoring[J].Anhui AgriculturalSciences ,2019,47(23):251-254.

6. Bruneau M,Chang $S$ E,Eguchi $R$ T,et al. A Framework to Quantitatively Assess and Enhance the Seismic Resilience of Communities[J].Earthquake spectra,2003,19(4): 733-752.

7. Han Jiannan, Li Yonghong, Liu Hainan, et al. Assessment of debris flow gully risk based on resilience of integrated control system [J].] Disaster science, 2018,33(1):230-234.

8. Shaw R, Razafindrab B. Climate Disaster Resilience: Focus On Coastal Urban Cities In Asia[M].Kyoto:The Asian Journal of Environment and Disaster Management, 2009: 4.

9. Chen C, Noble I, Hellmann J, et al. University of Notre Dame Global Adaptation Index CountryIndex Technical Report[J]. ND-GAIN: South Bend, IN, USA, 2015.

10. Zhang Hao, Li Xiangyang, Liu Zhaoge. Selection of CI resilience assessment indicators driven by case-data integration [J].] Science and technology for safe production in China,2018,14(4):13-19.

11. Shi Peijun, Yuan Yi. Comprehensive assessment of major natural disasters [J].] Advances in Geoscience ,2014,33(9):1145-1151.

12. Gu Hongbo, Tang Kai, Liu Xinyi, et al. Harm of Agricultural Flood and Drought Disaster and Construction of Defense System in China [J].] Journal of Hunan University of Science and
Technology
(Social
Sciences

Edition),2013,16(3):123-126.

13. Qin Zhihao, Xu Bin, Li Maosong, et al. Advances in Mechanism and Monitoring of Major Agrometeorological Disasters in China [J].] Journal of Natural Disasters ,2005,14(2):61-69.

14. Gu Hongbo, Liu Xinyi, Liu Zhiyu. Study on resilience assessment of agricultural drought and disaster based on variable fuzzy evaluation-Taking Hebei Province as an example [J]. Agricultural Research in Arid Areas ,2014,(5):174-180.

15. Zhang Xiangling, Li Xiaojian, Zhu Jiguang, et al. A Study on Spatial Differentiation of Development Level of Agricultural Modernization in Henan Province [J].] Geographical Research and Development ,2017,36(3):142-147.

16. Han Hong, Li Bo, Feng Guanghui. Time series change and spatial difference analysis of financial ecological operation quality-Empirical analysis based on financial ecology in Henan province [J].] Regional Research and Development ,2019,38(5):19-24. 